\title{
Single-Unit Response of Noradrenergic Neurons in the Locus Coeruleus of Freely Moving Cats. I. Acutely Presented Stressful and Nonstressful Stimuli
}

\author{
Elizabeth D. Abercrombie and Barry L. Jacobs \\ Program in Neuroscience, Department of Psychology, Princeton University, Princeton, New Jersey 08544
}

\begin{abstract}
The present experiment was designed to explore the stressrelatedness of activity in noradrenergic neurons of the locus coeruleus (LC) of behaving cats. A stressor was defined as a stimulus that elicited a significant sympathoadrenal activation as measured by plasma norepinephrine level and heart rate. According to this definition, exposure to $15 \mathrm{~min}$ of 100 dB white noise or 15 min of restraint was stressful in cats. In contrast, exposure to inaccessible rats for $15 \mathrm{~min}$ was behaviorally activating but nonstressful. The single-unit activity of noradrenergic neurons in the LC of behaving cats was examined under these conditions. The stressful stimuli elicited a significant increase in LC neuronal activity for the entire $15 \mathrm{~min}$ stressor duration, whereas the behaviorally activating but nonstressful stimulus elicited no significant change in the activity of these neurons. These results provide evidence that behavioral activation per se is not sufficient to evoke a tonic activation of these neurons. Rather, these data support the hypothesis that the LC is involved in the CNS response to stress and provide additional evidence that the activity of LC noradrenergic neurons increases in association with sympathoadrenal activation.
\end{abstract}

Various stressors increase biochemical indices of central norepinephrine (NE) utilization in extensive areas of the rat brain (e.g., Stone, 1975; Tsuda et al., 1982; Anisman et al., 1984). The locus coeruleus (LC), which contains as many as half of all $\mathrm{NE}$ neurons in the brain (Chu and Bloom, 1974; Jones and Moore, 1974; Wiklund et al., 1981), has been hypothesized to play an important role in this response (Korf et al., 1973; Cassens et al., 1980; Glavin, 1985; Weiss and Simson, 1985). Brain areas that receive their sole NE innervation from the LC show these changes during stress (Korf et al., 1973). A definitive relationship between stress and $\mathrm{LC}$ activation cannot readily be ascertained from neurochemical studies of this type, however, because of the indirect nature of the measurements employed and the lack of temporal contiguity between manipulation and measure. Therefore, in this and the following paper, we examine the nature of the relationship between stressful stimulation and activation of LC NE neurons by recording the single-unit activity of these neurons in freely moving cats.

Received Dec. 9, 1986; revised Mar. 23, 1987; accepted Mar. 24, 1987.

This work was supported by NIMH Predoctoral Fellowship Grant MH09256 (to E.D.A.) and NIMH Grant MH23433 and Air Force Grant AFOSR85-0034 (to B.L.J.).

Correspondence should be addressed to Dr. Abercrombie, Department of Behavioral Neuroscience, University of Pittsburgh, Pittsburgh, PA 15260.

Copyright (C) 1987 Society for Neuroscience $0270-6474 / 87 / 092837-07 \$ 02.00 / 0$
Previous electrophysiological studies of LC neurons in unanesthetized animals have shown that these neurons are activated by a wide variety of environmental stimuli of many modalities (Foote and Bloom, 1979; Foote et al., 1980; Aston-Jones and Bloom, 1981b; Rasmussen et al., 1986). In these studies, effective stimuli included flashes and clicks, which would be characterized as nonstressful. The neuronal responses to these phasic stimuli were, however, moderate ( 1 or 2 action potentials per stimulus) and short-lived (typically about $100 \mathrm{msec}$ ). On the other hand, no significant change in LC unit activity is seen in response to a variety of nonstressful tonic stimuli such as food presentation (German and Fetz, 1976; Rasmussen et al., 1986) or presentation of a conspecific (Rasmussen et al., 1986). Indeed, data from unanesthetized primates (Grant and Redmond, 1984) and cats (Rasmussen et al., 1986) suggest that the stimuli most capable of evoking tonic increases in LC unit activity are those that may be considered aversive or a challenge to the organism (i.e., stressors).

The conclusions in studies such as these are limited, however, in that the distinction between stressful and nonstressful stimuli employed were qualitative or subjective. The present studies attempt to overcome this limitation. It is well known that sympathoadrenal activation is an essential component of the physiological response to stress (Cannon, 1914; Mason et al., 1961; Levi, 1967). Therefore, we characterized the degree of sympathoadrenal activation elicited by a presumed nonstressful, but arousing, stimulus (exposure of the cats to inaccessible rats) and by aversive and presumed stressful stimuli (exposure to $100 \mathrm{~dB}$ white noise or restraint). This was accomplished by recording changes in heart rate and plasma NE levels elicited by these stimuli. The single-unit responses of LC neurons to these stimuli were then examined within this context, along with the temporal relationships between stressor, sympathoadrenal activation, and LC unit activity.

Additionally, the relationship between the degree of sympathoadrenal activation and the degree of activation of LC neurons elicited by these stimuli is of interest in its own right. It has been noted that brain NE neurons seem to share characteristics of the sympathetic ganglia both in terms of anatomical similarities and in terms of modulation of target cells. These observations have led to the proposal that the $\mathrm{LC}$ is a central analog of peripheral sympathetic ganglia (Amaral and Sinnamon, 1977; Aghajanian and VanderMaelen, 1982; Elam et al., 1986; Reiner, 1986).

The present study therefore examined the degree to which stimuli classified as stressors and nonstressors activate noradrenergic neurons of the $L C$, as well as the temporal relationships 
among stressors, sympathoadrenal activation, and LC unit activity. This study represents the first attempt to examine directly and systematically the relationship between a defined stress response and a change in brain single-unit activity in behaving animals. In the accompanying paper (Abercrombie and Jacobs, 1987), these results are extended to include an investigation of the nature of the relationship between LC unit activity and adaptation to stress.

\section{Materials and Methods}

Surgical procedure. Adult cats of either sex weighing between 2.5 and $4.0 \mathrm{~kg}(n=15)$ were anesthetized with sodium pentobarbital $(35 \mathrm{mg} /$ $\mathrm{kg}$, i.p.) and placed in a stereotaxic device. An incision was made along the midline on the dorsal surface of the head, the temporalis muscle was retracted, and the skull thoroughly cleaned. Two stainless steel screws for bipolar recording of the EEG were threaded through the skull at a location (A 27, L 10) that yields slow waves and sleep spindles during slow-wave sleep. Eye movements (EOG) were recorded by a stainless steel screw threaded into the frontal bone immediately posterior to the ocular orbit. EMG was recorded from the uninsulated tips of 2 stainless steel, multistranded wires inserted bilatcrally into the dorsal muscles of the neck. Another stainless steel screw was implanted in the frontal bone above the sinus to serve as a reference electrode. Finally, the cat was grounded by a stainless steel screw threaded into the parietal bone laterally so that the previously retracted temporalis muscle would cover it.

Following implantation of the gross electrodes, a burr hole was drilled to allow for the implantation of the microdrive assembly. This assembly consisted of 2 inner cannulae ( 23 gauge stainless steel tubing), separated by $1.0 \mathrm{~mm}$, that could be lowered through 2 outer guide cannulae (19 gauge stainless steel tubing) by means of an attached jeweler's screw $(0-80 \times 3 / 1$ inch). Using stereotaxic technique, the microdrive was positioned at a $30^{\circ}$ angle so that the tips of the inner cannulae were $6.0 \mathrm{~mm}$ dorsocaudal to the LC (A 0.6, H 15.5, L 2.8-3.0). The microdrive was cemented into this position with dental acrylic.

With the microdrive in place, 2 microelectrode bundles, each consisting of four $64 \mu \mathrm{m}$ and three $32 \mu \mathrm{m}$ Formvar-coated nichrome wires soldered to a 25 pin connector, were lowered through the guide cannulae to extend $5.0 \mathrm{~mm}$ past the guide cannulae tips. In this way, the microelectrodes were positioned $1.0 \mathrm{~mm}$ dorsocaudal to the $\mathrm{LC}$. The microelectrode bundles were glued to the tops of the inner cannulae after insertion. Thus, when the inner cannulae were advanced manually (in 40 or $80 \mu \mathrm{m}$ increments) by means of the attached jeweler's screw, the microelectrodes were advanced through the brain. At the time of surgery, some animals also received an indwelling chronic i.v. catheter for sampling plasma.

Recording procedure. Following a postoperative recovery period of approximately $7 \mathrm{~d}$, cats were habituated to an electrically shielded, sound-attenuating recording chamber $(65 \times 65 \times 95 \mathrm{~cm}$ with a clear plexiglass door). Electrical signals were led from the animal via a counter-weighted, low-noise shielded cable and commutator to EEG amplifiers (Grass 7P511) or polygraph amplifiers for recording of unit potentials and gross potentials, respectively. For the recording of unit potentials, a second microelectrode in the same bundle served as the indifferent electrode. Unit potentials were monitored on-line with the use of a storage oscilloscope. Single-unit activity was separated from background noise by a variable threshold gate-Schmitt trigger. The pulse output from the Schmitt trigger was used to produce a click through a small speaker, a polygraph pen deflection, and inputs to an electronic nerve spike counter. Additionally, the EKG was recorded by means of subdermal electrodes placed bilaterally on the rib cage of the animal prior to the beginning of the recording session. EEG, EOG, EMG, and EKG potentials were written out on-line on polygraph paper. During the experimental session, the behavior of the animal was continuously monitored via video camera, and behavioral observations were noted on the polygraph paper simultaneously with the outputs of the unit and gross potential channels.

Presumed noradrenergic units were initially identified on-line by the following criteria: (1) a long-duration action potential (>2.0 msec), (2) slow (about $1.0 \mathrm{spike} / \mathrm{sec}$ in quiet waking) pattern of discharge, and (3) complete suppression of activity during rapid eye movement sleep. These characteristics have been observed in LC neurons of both the behaving rat (Aston-Jones and Bloom, 1981a) and cat (Rasmussen et al., 1986). At the end of a recording session, the neurochemical identity of the neurons studied was further confirmed by administering the $\alpha$, agonist clonidine hydrochloride $(25 \mu \mathrm{g} / \mathrm{kg}$, i.p.), which has been reported to cause a significant suppression of NE units within $30 \mathrm{~min}$ of injection (Reiner, 1985; Rasmussen et al., 1986). In addition, the recording sites of the neurons studied were histologically localized to the area of the LC.

Since, in the cat, NE neurons in the area of the LC are interspersed among non-NE neurons, there is an issue of neurochemical identification of the cells under study. Because the cells examined in behaving animals cannot be marked intracellularly, the recorded cell cannot subsequently be localized and then identified as NE by fluorescence- or immunohistochemical staining procedures. However, the characteristics described above represent a distinctive constellation and are observed only from recording sites in the area of the LC (this issue has not yet been explored in other groups of brain NE neurons in behaving animals). Thus, although it is presently not possible in single-unit studies in behaving cats to directly identify cells in the LC as NE, their identification, based on a number of indirect criteria, seems clear-cut.

Unit recordings. Cells in the area of the LC fitting all criteria for identification as noradrenergic were randomly assigned to one of 3 conditions: inaccessible rats, white noise, or restraint. Baseline data corresponding to quiet waking (QW) and active waking (AW) behavioral states were collected. These behavioral states were defined according to polygraphic and behavioral criteria previously published (Trulson and Jacobs, 1979). The animal was left undisturbed for at least $15 \mathrm{~min}$ before the onset of the experimental stimulus. Animals were never exposed to the same stressor more than once, although some animals participated in more than one condition.

Inaccessible rats. A pair of adult albino rats was placed in a clear plexiglass rat cage $(45 \times 20 \times 23 \mathrm{~cm})$ with a wire mesh lid and containing approximately $2 \mathrm{~cm}$ of wood shavings. The cage was placed into the recording chamber with the cat and remained there for $15 \mathrm{~min}$. Unit activity and heart rate were monitored during this time and for $15 \mathrm{~min}$ after removal of the rats.

White noise. White noise of $100 \mathrm{~dB}$ intensity was produced via a speaker located in one wall of the recording chamber. Unit activity and heart rate were recorded continuously for $15 \mathrm{~min}$ during the stimulus, as well as for 15 min after noise offset.

Restraint. Restraint stress was effected by placing the cat in a commercially available canvas handling bag $(18 \times 9 \times 6$ inch $)$ and then wrapping a $5 \mathrm{ft} \times 6$ inch length of elastic bandage around the bag to conform to the size of the individual animal. Movement of the limbs was precluded by this procedure while leaving the head free to move. The restraint was applied for $15 \mathrm{~min}$, and both unit activity and heart rate were recorded continuously for the entire stress period, as well as for 15 min following the termination of restraint.

Plasma NE determination. Changes in plasma NE levels in response to inaccessible rats, white noise, or restraint were determined in a separate group of cats independently of the changes in unit activity elicited by these stimuli. This was necessitated because the effective life of the i.v. catheters for withdrawal of blood was found to be relatively short compared with the time between electrode implantation and unit recording (typically 2 weeks to scveral months). Not all animals were tested with all stimuli.

Blood was collected $15 \mathrm{~min}$ prior to stimulus onset, 1 and $15 \mathrm{~min}$ into the stimulus, and $15 \mathrm{~min}$ after stimulus offset. Blood sampling was accomplished by the experimenter entering the recording chamber and drawing a $2.0 \mathrm{ml}$ blood sample. The samples were immediately centrifuged at $2500 \mathrm{rpm}$ for $10 \mathrm{~min}$. The plasma was then drawn off and frozen at $-80^{\circ} \mathrm{C}$ until assay. Heart rate was recorded continuously during all blood sampling procedures.

NE levels in plasma were determined using high-pressure liquid chromatography (HPLC) with electrochemical detection (Hallman et al., 1978).

Data analysis. The plasma NE values obtained from the HPLC analysis (expressed as $\mathrm{pg} / \mathrm{ml}$ plasma) were analyzed using ANOVA with repeated measurcs for cach condition. Post hoc tests were used to evaluate the significance of difference from prestimulus baseline.

Similarly, the heart rate response (beats/min) at corresponding time points in each condition was evaluated using ANOVA with repealed measures and post hoc tests. This analysis was carried out both for heart rate data obtained during plasma sampling and during unit recording.

The Pearson correlation coefficient was determined for the plasma $\mathrm{NE}$ and heart rate response or the NE unit and heart rate response in 



Figure 1. Plasma NE (solid bars) and accompanying heart rate (circles) responses to inaccessible rats (left, $n=7)$, white noise (center, $n=7)$, and restraint (right, $n=7$ ). Data shown are means \pm SEM. *, significantly greater than baseline $(p<0.05)$. $B L$, baseline; 1 , minute 1 of manipulation; 15 , minute 15 of manipulation; $P, 15 \mathrm{~min}$ postmanipulation.

each animal. These within-subject analyses were carried out to examine the relationship between these variables independent of interindividual variability.

Unit data were analyzed by first calculating the mean discharge rate of the cells studied during AW, and during minutes $1,5,10$, and 15 , as well as $15 \mathrm{~min}$ after experimental manipulations (obtained from 6 consecutive $10 \mathrm{sec}$ time samples). These values (expressed as spikes/ sec \pm SEM) were then used to assess the effect of the experimental conditions on discharge rate with one-way ANOVA with repeated measures and post hoc tests to compare firing rates to baseline AW periods. In addition, the 6 consecutive $10 \mathrm{sec}$ counts obtained during baseline and during the above time points were examined on an individual cell basis using one-way analysis of variance with post hoc tests. This was done to obtain a more detailed analysis of the effect of the stimuli on these neurons. The level of significance for all statistical analyses in the present experiments was set at $p<0.05$.

\section{Results}

\section{Plasma NE and accompanying heart rate responses}

Inaccessible rats. The plasma NE response to the $15 \mathrm{~min}$ presentation of inaccessible rats was examined in 7 cats (Fig. 1, left). In these cats, no significant differences from baseline levels were detected at any time $[F(3,18)=2.13, p=0.132]$, although a small $9 \%$ increase was observed in minute 1 .

Analysis of the heart rate in these animals showed no significant change in response to exposure to inaccessible rats $[F(3,18)=3.71, p=0.052]$ (Fig. 1, left). Heart rate was $154 \pm$ 14 at baseline and $159 \pm 13$ during the first minute.

White noise. Analysis of plasma samples obtained from 7 cats during a single $15 \mathrm{~min}$ presentation of white noise revealed that this stimulus evoked a significant increase in plasma NF levels $[F(3,18)=15.80, p<0.0001]$ (Fig. 1, center). The increase was significantly greater than baseline at both 1 and 15 min during the stimulus, showing elevations of 146 and $100 \%$, respectively. Plasma NE 15 min after the noise stimulus was no longer significantly different from baseline ( $47 \%$ above baseline).

Consistent with these results, heart rate was also significantly elevated by white noise $[F(3,18)=30.60, p<0.0001]$ (Fig. 1, center). At 1 and $15 \mathrm{~min}$ during the noise, heart rate showed significant elevations from a baseline level of $143 \pm 9$ beats/ $\min$ to $213 \pm 13$ and $198 \pm 15$ beats $/ \mathrm{min}$, respectively. By 15 min after noise offset, heart rate $(160 \pm 9$ beats $/ \mathrm{min})$ was no longer significantly elevated.

Restraint. Similarly, a significant increase in plasma NE was found in 7 cats in response to $15 \mathrm{~min}$ of restraint $[\mathrm{F}(3,18)=$ 18.4, $p<0.0001 ; n=7$ ] (Fig. 1, right). Plasma NE was significantly greater than baseline at minutes $1(137 \%)$ and 15 (105\%) of the stimulus. By 15 min poststimulus, plasma NE had returned to near baseline levels ( $8 \%$ over baseline).

Likewise, heart rate was significantly elevated by restraint $[F(3,18)=79.06, p<0.0001]$ (Fig. 1, right). At 1 and $15 \mathrm{~min}$ during the stimulus, heart rate was $221 \pm 5$ and $211 \pm 7$ beats/ min, respectively, compared with the baseline level of $156 \pm 9$ beats/min. By 15 min postrestraint, heart rate (168 \pm 7 beats/ min) was no longer significantly different from baseline.

The Pearson correlation coefficients for plasma NE level and heart rate for the response of each animal in each group showed the 2 variables to be highly positively correlated during the time points examined, especially for the white noise and restraint conditions. The means of these values were $r=0.45$ for inaccessible rats, $r=0.89$ for white noise, and $r=0.97$ for restraint (see Fig. 1).

\section{LC unit activity, behavior, and accompanying heart rate responses}

Inaccessible rats. During the 15 min presentation of inaccessible rats, the cats typically remained focused on the rats as evidenced by an orientation of the body toward the rats and sniffing and pawing at their cage. The cats often attempted to gain access to the rats by pawing or striking at them or by climbing on top of the rat cage and attempting to reach through the wire mesh. This behavioral activation was further documented by a lowvoltage, fast EEG and frequent phasic changes in EOG and EMG activity (Fig. 2). The responses of $7 \mathrm{NE}$ cells were recorded during presentation of inaccessible rats, as were the accompanying heart rate responses.

A signficant overall effect of inaccessible rats on unit activity was found $[F(6,36)=6.25, p=0.007]$; however, no individual time point examined was significantly greater than baseline (Fig. 3 , top). Indeed, mean unit activity 15 min after presentation $(0.7 \pm 0.1 \mathrm{spikes} / \mathrm{sec})$ was significantly lower than AW baseline, accounting for the significant overall effect. With post hoc tests, mean unit activity during minutes $1,5,10$, and 15 , as well as 15 min poststimulus, was compared with $\mathrm{AW}$. Mean unit activity was highest during the first minute of the presentation of the rats $(1.3 \pm 0.1$ spikes/sec) but was not significantly greater than the AW baseline level $(1.1 \pm 0.1$ spikes/sec $)$ at any time.

Paralleling the lack of an $\mathrm{LC}$ unit response to inaccessible rats, there were no significant changes in the accompanying heart rate $[F(6,36)=2.30, p=0.10]$, although small increases were observed at several time points (Fig. 3 , bottom). The mean value of the Pearson correlation coefficients for LC unit acitivity and heart rate during inaccessible rats was $r=0.24$.

White noise. The NE unit response to $15 \mathrm{~min}$ of $100 \mathrm{~dB}$ white noise was examined in 7 cells along with the concomitant effects upon heart rate. The behavioral response most commonly elicited by this stimulus included flattening of the ears and a crouched posture, with little overt motor activity. The EEG was char- 
INACCESSIBLE RATS
UNIT

EEG
RESTRAINT

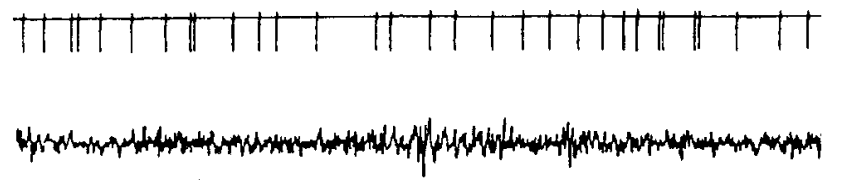

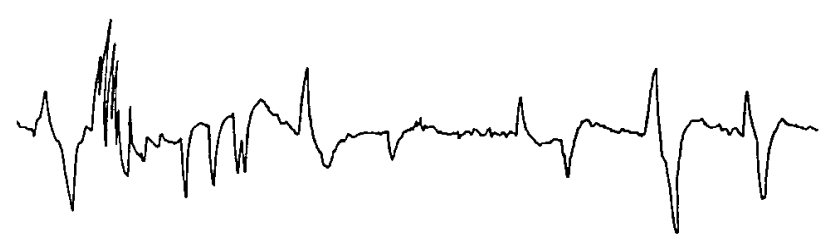
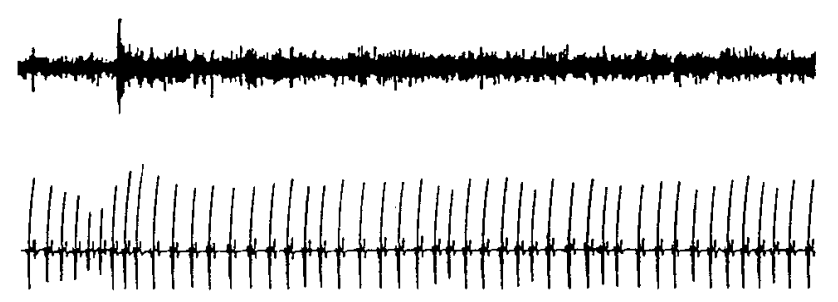

$E O G$

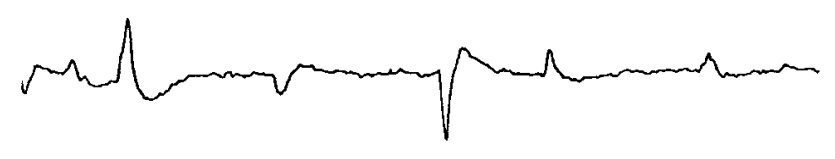

EKG

EMG

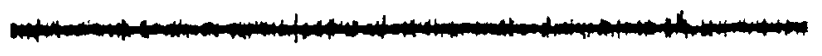

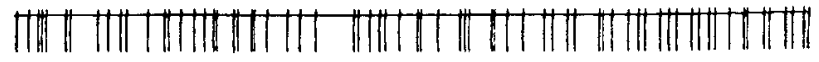

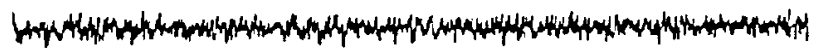

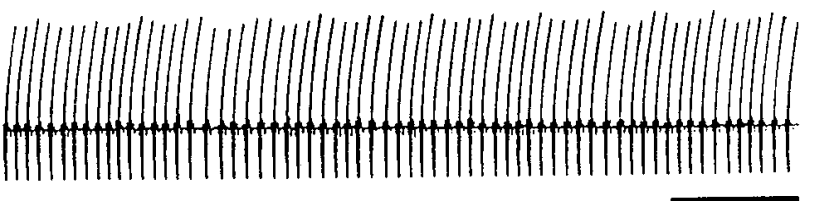

$5 \mathrm{sec}$

Figure 2. Polygraph record $(30 \mathrm{sec})$ comparing the firing rate of the same LC NE neuron and accompanying EEG, EOG, EMG, and EKG at approximately $5 \mathrm{~min}$ into inaccessible rats and restraint. The unit firing rate (in spikes/sec) was 1.0 during inaccessible rats and 2.3 during restraint. The EKG indicates a greater degree of sympathetic activation during restraint; heart rate (in beats/min) was 94 during inaccessible rats and 130 during restraint.

acterized by low-voltage, fast activity throughout the stimulus presentation.

White noise produced a highly significant increase in NE unit activity $[F(6,36)=10.47, p<0.0001]$ (Fig. 4, top). Compared with AW baseline, unit activity was significantly elevated at minutes $1,5,10$, and 15 . By 15 min poststimulus, unit activity returned to baseline levels. During the first minute of the stimulus, mean unit firing rate reached $2.4 \pm 0.3$ spikes $/ \mathrm{sec}$ compared with the $\Lambda \mathrm{W}$ level of $1.4 \pm 0.2$ spikes/sec and returned to $1.1 \pm 0.2 \mathrm{spikes} / \mathrm{sec} 15 \mathrm{~min}$ after. The individual cell analysis of the LC unit responses to white noise revealed that each of the cells studied was significantly activated by this stimulus.

An examination of the six $10 \mathrm{sec}$ unit activity counts obtained during the first minute of white noise and the first minute after white noise offset was revealing. (Only white noise was studied in this way since discrete onset and offset points could not be determined for restraint.) When the noise began, $L C$ unit activity showed an immediate increase in the first $10 \mathrm{sec}$, which had declined by only $11 \%$ at the end of minute 1 . Over the $15 \mathrm{~min}$ course of white noise, unit activity declined slightly from the maximum 2.4 spikes/sec during minute 1 to 2.0 spikes/sec during minute 15. Upon termination of the noise, LC unit activity gradually decreased over the course of the first minute, bringing activity to near AW levels (1.7 and $1.4 \mathrm{spikes} / \mathrm{sec}$ for $1 \mathrm{~min}$ after noise offset and $\mathrm{AW}$, respectively).

The concomitant changes in heart rate were likewise highly significant $[F(6,36)=6.33, p=0.0001]$ (Fig. 4, bottom). Heart rate was significantly elevated at minutes $1,5,10$, and 15 during the white noise. Heart rate during minute 1 was $171 \pm 10$ beats/ min compared with a baseline level of $135 \pm 9$ beats $/ \mathrm{min}$. This elevation was no longer apparent by $15 \mathrm{~min}$ poststimulus.

LC unit activity and heart rate strongly covaried in response to white noise. The mean Pearson correlation coefficient for this condition was $r=0.77$.

Restraint. The effects of 15 min of restraint on LC unit activity were examined in 7 cells. The parallel changes in heart rate were also studied. During restraint, the EEG was composed of lowvoltage, fast activity, and the cats typically remained quite still with occasional intermittent episodes of struggling and vocalization (Fig. 2).

NE neurons responded to this manipulation with a highly significant elevation of activity $[F(6,36)=8.91, p<0.0001]$ (Fig. 5, top). The observed increase significantly differed from AW baseline at minutes $1,5,10$, and 15 , returning to baseline 15 min aftcr relcasc. The mean AW level of activity in this condition was $1.4 \pm 0.3$ spikes/sec compared with $2.7 \pm 0.5$ spikes/sec during the first minute of restraint and $1.0 \pm 0.2$ spikes/sec by $15 \mathrm{~min}$ after restraint. Analysis of the individual unit responses to restraint revealed a significant activation in all 7 neurons studied.

Similarly, the heart rate response to $15 \mathrm{~min}$ of restraint was significantly elevated $[F(6,36)=7.97, p<0.0001]$ (Fig. 5 , bottom). This increase was evident at minutes $1,5,10$, and 15 . Heart rate was $197 \pm 16$ beats/min during minute 1 as compared to a $141 \pm 13$ beats $/ \mathrm{min}$ baseline. Heart rate was no longer significantly elevated $15 \mathrm{~min}$ after restraint. The mean value of the Pearson correlation coefficient for LC unit activity and heart rate during restraint was $r=0.57$. 
INACCESSIBLE RATS
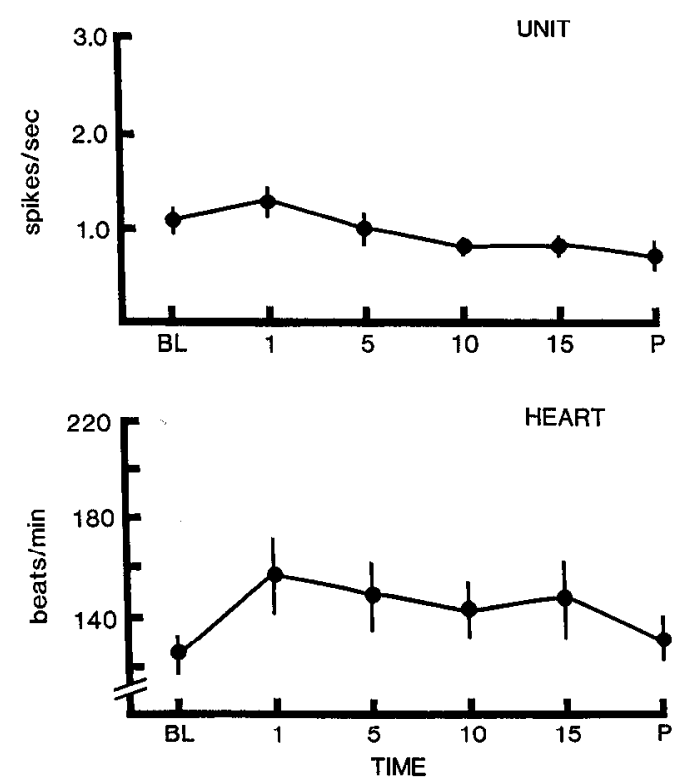

Figure 3. Summary of LC NE unit (top) and accompanying heart rate (bottom) responses during inaccessible rats $(n=7)$. Data shown are means \pm SEM. *, significantly greater than baseline $(p<0.05) . B L$, baseline; $l$, minute 1 of manipulation; 5 , minute 5 of manipulation; 10 , minute 10 of manipulation; 15 , minute 15 of manipulation; $P, 15$ min postmanipulation.

\section{Discussion}

The purpose of examining plasma NE and heart rate in this study was to establish the degree to which the experimental conditions were capable of eliciting a quantifiable stress response in conscious cats. Since the majority of studies on the neurobiology of stress have been conducted in other species, this was a necessary foundation for the subsequent examination of the stress-relatedness of feline LC neuronal activity. It was found that exposure of cats to inaccessible rats, although behaviorally activating, could not be considered a stressor as defined by a significant elevation of heart rate and plasma NE. In contrast, exposure to $100 \mathrm{~dB}$ white noise and restraint evoked significant increases in both heart rate and plasma NE levels that were sustained for the 15 min duration of the stimuli. On the basis of these results, these latter two stimuli were classified as stressors under the conditions of the present experiment.

Heart rate and plasma NE displayed highly correlated changes in response to both stressors. On this basis, it could be argued that, at least for the conditions studied, a significant tonic increase in heart rate has a high probability of being accompanied by a significant increase in plasma NE levels. Stated another way, the high correlation between heart rate and plasma NE levels was used to validate the use of heart rate alone as an index of the stress response in the examination of LC unit activity. Here, it was also found that inaccessible rats failed to elicit a significant increase in heart rate and that the white noise and restraint procedures were effective in this respect. Based on these observations it is concluded that, in conscious cats, exposure to inaccessible rats is behaviorally activating but nonstressful, whereas exposure to $100 \mathrm{~dB}$ white noise or restraint is indeed stressful. It is also interesting that the correlation between heart rate and plasma NE was much lower during pre-
WHITE NOISE
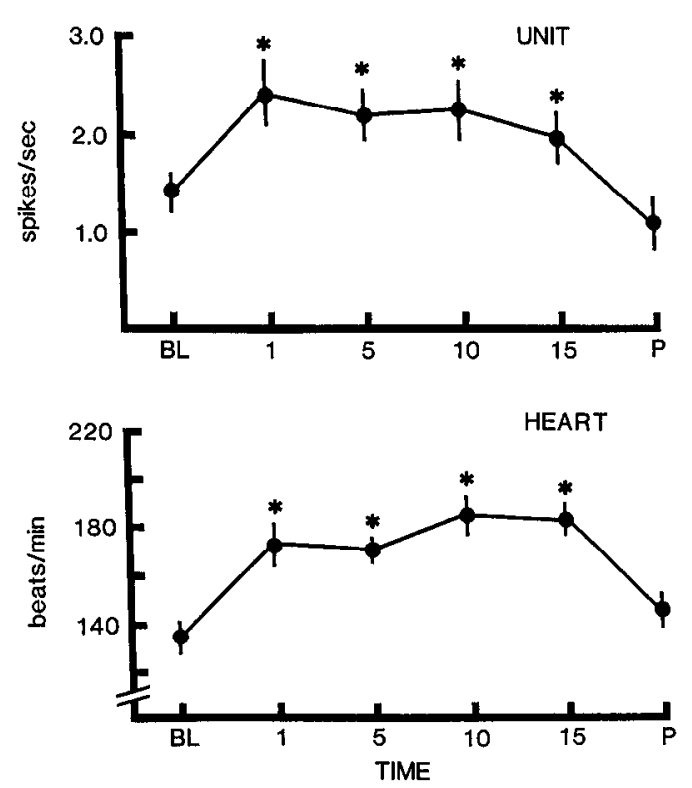

Figure 4. Summary of LC NE unit (top) and accompanying heart rate (bottom) responses during $100 \mathrm{~dB}$ white noise $(n=7)$. Data shown are means \pm SEM. *, significantly greater than baseline $(p<0.05)$. See Figure 3 for explanation of abbreviations.

sentation of the inaccessible rats. This may be a reflection of somewhat random fluctuations that occur in physiological systems that are in a nonactivated state.

This study is the first to examine the physiological response parameters of cats to various environmental stimuli and one of the few studies in which basal plasma NE concentrations have
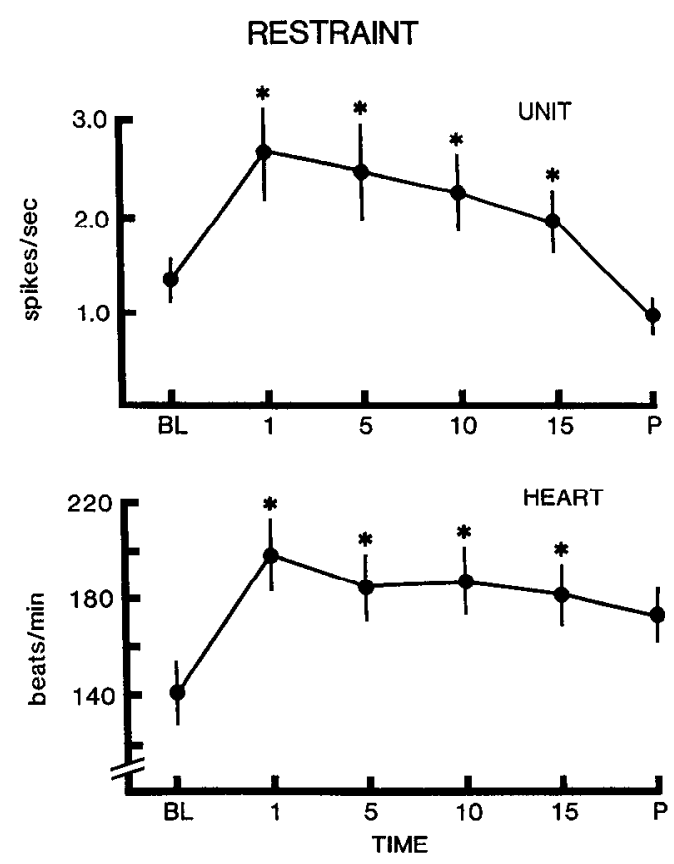

Figure 5. Summary of LC NE unit (top) and accompanying heart rate (bottom) responses during restraint $(n=7)$. Data shown are means \pm SEM. *, significantly greater than baseline $(p<0.05)$. See Figure 3 for explanation of abbreviations. 
been measured in conscious cats. The magnitude of stress-induced changes reported is in general agreement with those obtained using other species. Studies employing immobilization in rats report 4- to 6-fold increases in plasma NE (Popper et al., 1977). The approximatcly 3 -fold increase in plasma NE in response to restraint in the present study is slightly lower, perhaps as a result of differences in the quality of the stimulus or of the fact that, since the experimenter entered the recording chamber, the blood sampling procedure may have been mildly arousing, thus elevating baseline values. In support of this latter interpretation, a study by Hubbard and colleagues (1986) using adapted cats in isolation chambers reported basal plasma NE values of $195 \pm 18 \mathrm{pg} / \mathrm{ml}$ as compared with $868 \pm 198 \mathrm{pg} / \mathrm{ml}$ in the present study. On the other hand, Buhler et al. (1978) reported basal plasma NE levels in the cat very similar to ours $(609 \pm 119 \mathrm{pg} / \mathrm{ml})$. Clearly, various factors are capable of affecting sympathoadrenal activity at a given moment, and care must be taken to minimize disturbance during blood collection.

The primary purpose of the present study was to examine the stress-relatedness of LC NE neurons. Having established that white noise and restraint were indeed stressful stimuli under the present conditions and that exposure to inaccessible rats was not, this issue was examined directly. LC units responded to both white noise and restraint with significant tonic increases in firing rate. Furthermore, this responsiveness was extremely homogeneous, occurring in all LC NE cells examined. This supports previous theories of LC function, in which this NE system is viewed as a rather homogeneous ensemble (cf. Aston-Jones, 1985). The increase in LC unit activity occurred immediately upon onset of the stressor, indicating that there is little or no inertia in this system. The increased unit activity was sustained with only a small decrement across the entire 15 min stress period. This finding significantly expands the known dynamic range of activity of $\mathrm{LC} \mathrm{NE}$ neurons. The nature and magnitude of the decrement in unit activity that accompanies exposure to prolonged or repeated stressors is examined in detail in the following paper (Abercrombie and Jacobs, 1987). In contrast to effects of stressors, the firing rate of LC neurons of cats presented with inaccessible rats did not exceed the level observed during AW despite the high degree of behavioral activation. This latter finding is in agreement with Rasmussen et al. (1986), who also found a slight, but nonsignificant, increase in the activity of LC neurons in freely moving cats exposed to inaccessible rats. This same study also reported no significant changes in LC unit activity in response to other nonnoxious, behaviorally activating stimuli such as exposure to inaccessible food, presentation of a conspecific, and walking on a treadmill.

It is interesting to note the behavioral responses of the cats during the 3 experimental conditions. During the 2 stressors, the cats remained alert as judged by the EEG record but seldom displayed any overt behaviors, as ascertained both by direct observation and EOG and EMG recordings. Epochs of struggling and vocalization were recorded during restraint, but these were brief compared with the large amount of time during which the animals were quiescent. Exposure to inaccessible rats elicited a qualitatively different pattern. During this time, the cats displayed a high degree of activation, as manifested behaviorally and in phasic changes in EOG and EMG activity. Thus, a dissociation between overt behavioral activation and increases in LC unit activity was found since exposure to inaccessible rats had little effect on unit activity. These behavioral observations, coupled with the data on the LC unit responses to the experi- mental manipulations, support the hypothesis that LC neurons are highly sensitive to stressful stimuli. Thus, an important finding of the present study is that behaviorally activating stimuli per se do not appear to be sufficient for tonic stimulation of this system above an AW baseline level, although behavioral activation may be an important component of the response to some stressful stimuli. Thus, arousal level is probably not the most reliable index of neuronal activity in this cell group.

A notable characteristic of the present data was the degree to which LC unit activity and sympathetic activation covaried. The results of the present study, taken together with the results of previous studies (Elam et al., 1984; Reiner, 1986), indicate that the activity of LC neurons does indeed increase in association with sympathetic activation. Only those stimuli that elicited a significant sympathetic activation produced a sustained elevation in LC unit activity. Furthermore, LC unit activity and heart rate were found to be strongly correlated during white noise and restraint. Generally, the greater the sympathetic activation in response to the stressor, as indicated by heart rate, the greater the correlation observed. Thus, a stimulus intensity threshold for coactivation of central and peripheral NE systems appears to exist. Stimuli of sufficient intensity (e.g., white noise and restraint) elicit an activation of LC neurons that parallels that of the peripheral NE system. With weaker stimuli, however, the activity of LC neurons is independent of the peripheral NE system; during exposure to inaccessible rats, LC unit activity and heart rate showed no consistent correlation. This relationship between LC neuronal activity and the sympathoadrenal system appears to generalize beyond the response to environmental stimuli, as similar results have been obtained studying the response of LC units in unanesthetized cats to several physiological challenges, including hypotension and hypoglycemia (Morilak et al., 1987a, b).

Finally, despite evidence implicating brain serotonergic neurons and dopaminergic neurons in the response to stress (e.g., Kennett and Joseph, 1981; Keller et al., 1983), it appears that a stress-related activation of monoaminergic systems may be somewhat specific to NE neurons. Previous studies utilizing the same or similar stimuli failed to obtain any significant activation of either serotonergic neurons of the dorsal raphe nucleus (Wilkinson and Jacobs, 1986) or dopaminergic neurons of the substantia nigra (Strecker and Jacobs, 1985). Dopaminergic neurons of the ventral tegmental area have not, however, been examined under these conditions.

\section{Summary}

The present experiment is the first to examine the activity of brain neurons during defined conditions of stress in behaving animals. We have shown that an increase in LC unit activity does indeed occur in response to stressors, as had been suggested by the neurochemical literature. This increase was sustained for the duration of the stressor and the changes were temporally related to stimulus onset and offset. Further, we have shown that a stimulus that simply increases behavioral activation in the absence of a physiological stress response is insufficient to sustain an activation of these neurons above the level observed during $\mathrm{AW}$. The most robust tonic activation of $\mathrm{LC}$ neurons under normal physiological conditions appears to occur during stress.

Based on these results, the following prediction can be made: As adaptation to a stressful stimulus occurs, as measured by the physiological stress response, the LC response to the stimulus 
should likewise adapt. This prediction is tested in the following paper (Abercrombie and Jacobs, 1987).

\section{References}

Abercrombie, E. D., and B. L. Jacobs (1987) Single-unit responsc of noradrenergic neurons in the locus coerulus of freely moving cats. II. Adaptation to chronically presented stressful stimuli. J. Neurosci. 7: 2844-2848.

Aghajanian, G. K., and C. P. VanderMaelen (1982) $\alpha_{2}$-Adrenoceptormediated hyperpolarization of locus coeruleus neurons: Intracellular studies in vivo. Science 215: 1394-1396.

Amaral, D. G., and H. M. Sinnamon (1977) The locus coeruleus: Neurobiology of a central noradrenergic nucleus. Prog. Neurobiol. 9: 147-196.

Anisman, H., L. Kokkinidis, and L. S. Sklar (1984) Neurochemical consequences of stress: Contributions of adaptive processes. In Psychological and Physiological Interaction in Response to Stress, S. Burchfield, ed., pp. 67-98, Hemisphere, New York.

Aston-Joncs, G. (1985) Bchavioral functions of locus coeruleus derived from cellular attributes. Physiol. Psychol. 13: 118-126.

Aston-Jones, G., and F. E. Bloom (1981a) Activity of norepinephrinecontaining locus coeruleus neurons in behaving rats anticipates fluctuations in the sleep-waking cycle. J. Neurosci. 1: 876-886.

Aston-Jones, G., and F. E. Bloom (1981b) Norepinephrine-containing locus coeruleus neurons in behaving rats exhibit pronounced responses to non-noxious environmental stimuli. J. Neurosci. $1: 887-$ 900.

Buhler, H. U., M. Da Prada, W. Haefely, and G. B. Picotti (1978) Plasma adrenaline, noradrenaline and dopamine in man and different animal species. J. Physiol. (Lond.) 276: 311-320.

Cannon, W. B. (1914) The emergency function of the adrenal medulla in pain and in the major emotions. Am. J. Physiol. 33: 356.

Cassens, G., M. Roffman, A. Kuruc, P. J. Orsulak, and J. J. Schildkraut (1980) Alterations in brain norepinephrine metabolism induced by environmental stimuli previously paired with inescapable shock. Science 209: 1138-1140.

Chu, N., and F. E. Bloom (1974) The catecholamine-containing neurons in the cat dorsolateral pontine tegmentum: Distribution of the cell bodies and some axonal projections. Brain Res. 66: 1-21.

Elam, M., T. Yao, T. H. Svensson, and P. Thoren (1984) Regulation of locus coeruleus neurons and splanchnic, sympathetic nerves by cardiovascular afferents. Brain Res. 290: 281-287.

Elam, M., T. H. Svensson, and P. Thoren (1986) Locus coeruleus neurons and sympathetic nerves: Activation by cutaneous sensory afferents. Brain Res. 366: 254-261.

Foote, S. L., and F. E. Bloom (1979) Activity of norepinephrinecontaining locus coeruleus neurons in the unanesthetized squirrel monkey. In Catecholamines: Basic and Clinical Frontiers, Vol. 1, E. Usdin, I. J. Kopin and J. Barchas, eds., pp. 625-627, Pergamon, New York.

Foote, S. L., G. Aston-Jones, and F. E. Bloom (1980) Impulse activity of locus coeruleus neurons in awake rats and monkeys is a function of sensory stimulation and arousal. Proc. Natl. Acad. Sci. USA 77: 3033-3037.

German, D. C., and E. E. Fetz (1976) Responses of primate locus coeruleus and subcoeruleus neurons to stimulation at reinforcing brain sites and to natural reinforcers. Brain Res. 109: 497-514.

Glavin, G. B. (1985) Stress and brain noradrenaline: A review. Neurosci. Biobehav. Rev. 9: 233-243.

Grant, S. J., and D. E. Redmond, Jr. (1984) Neuronal activity of the locus ceruleus in awake Macaca arctoides. Exp. Neurol. 84: 701-708.

Hallman, H., L.-O. Farnebo, B. Hamberger, and G. Jonsson (1978) A sensitive method for the determination of plasma catecholamines using liquid chromatography with electrochemical detection. Life Sci. 23: $1049-1052$.
Hubbard, J. W., R. A. Buchholz, T. K. Keeton, and M. A. Nathan (1986) Plasma norepinephrine concentration reflects pharmacological alteration of sympathetic activity in the conscious cat. J. Autonom. Nerv. Syst. 15: 93-100.

Jones, B. E., and R. Y. Moore (1974) Catecholamine-containing neurons of the nucleus locus coeruleus in the cat. J. Comp. Neurol. 157: 43-51.

Keller, R. W., E. M. Stricker, and M. J. Zigmond (1983) Environmental stimuli but not homeostatic challenges produce apparent increases in dopaminergic activity in the striatum: An analysis by in vivo voltammetry. Brain Res. 279: 159-170.

Kennett, G. A., and M. H. Joseph (1981) The functional importance of increased brain tryptophan in the serotonergic response to restraint stress. Neuropharmacology 20: 39-43.

Korf, J., G. K. Aghajanian, and R. H. Roth (1973) Increased turnover of norepinephrine in the rat cerebral cortex during stress: Role of the locus coeruleus. Neuropharmacology 12: 933-938.

Levi, L. (1967) Stressors, stress tolerance, emotions and performance in relation to catecholamine excretion. In Emotional Stress, L. Levi, ed., pp. 192-200, Elscvicr, New York.

Mason, J. W., G. Mangan, Jr., J. V. Brady, D. Conrad, and D. McK. Rioch (1961) Concurrent plasma epinephrine, norepinephrine and 17-hydroxycorticosteroid levels during conditioned emotional disturbance in monkeys. Psychosomat. Med. 23: 344-353.

Morilak, D. A., C. A. Fornal, and B. L. Jacobs (1987a) Effects of physiological manipulations on locus coeruleus neuronal activity in freely moving cats: II. Cardiovascular challenge. Brain Res. (in press).

Morilak, D. A., C. A. Fornal, and B. L. Jacobs (1987b) Effects of physiological manipulations on locus coeruleus neuronal activity in freely moving cats: III. Glucoregulatory challenge. Brain Res. (in press).

Popper, C. W. C. C. Chiueh, and I. J. Kopin (1977) Plasma catecholamine concentrations in unanesthetized rats during sleep, wakefulness, immobilization and after decapitation. J. Pharmacol. Exp. Ther. 202: 144-148.

Rasmussen, K., D. A. Morilak, and B. L. Jacobs (1986) Single unit activity of locus coeruleus neurons in the freely moving cat. I. During naturalistic behaviors and in response to simple and complex stimuli. Brain Res. 371: 324-334.

Reiner, P. B. (1985) Clonidine inhibits central noradrenergic neurons in unanesthetized cats. Eur. J. Pharmacol. 115: 249-257.

Reiner, P. B. (1986) Correlational analysis of central noradrenergic neuronal activity and sympathetic tone in behaving cats. Brain Res. 378: 86-96.

Stone, E. A. (1975) Stress and catecholamines. In Catecholamines and Behavior, Vol. 2, A. J. Friedhoff, ed., pp. 31-72, Plenum, New York.

Strecker, R. E., and B. L. Jacobs (1985) Substantia nigra dopaminergic unit activity in behaving cats: Effect of arousal on spontaneous discharge and sensory evoked activity. Brain Res. 361: 339-350.

Trulson, M. E., and B. L. Jacobs (1979) Raphe unit activity in freely moving cats: Correlation with level of behavioral arousal. Brain Res. 163: 135-150.

Tsuda, A., M. Tanaka, Y. Kohno, T. Nishikawa, K. Iimori, R. Nakagawa, Y. Hoaki, Y. Ida, and N. Nagasaki (1982) Marked enhancement of noradenaline turnover in extensive brain regions after activity-stress in rats. Physiol. Behav. 29: 337-341.

Weiss, J. M., and P. G. Simson (1985) Neurochemical basis of stressinduced depression. Psychopharm. Bull. 21: 447-457.

Wiklund, L., L. Leger, and M. Persson (1981) Monoamine cell distribution in the cat brainstem. A fluorescence histochemical study with quantification of indolaminergic and locus coeruleus cell groups. J. Comp. Neurol. 203: 613-647.

Wilkinson, L. O., E. D. Abercrombie, and B. L. Jacobs (1986) Environmental stress and activity of dorsal raphe serotonergic neurons in the freely moving cat. Soc. Neurosci. Abstr. 12: 1134. 